\title{
Drug guest molecules as modulators during one-step mechanochemical encapsulation in MIL-100 (Fe) framework
}

\author{
Barbara E. Souza, ${ }^{[a]}$ Annika F. Möslein, ${ }^{[a]}$, Kirill Titov ${ }^{[a]}$ James D. Taylor, ${ }^{[b]}$ Svemir Rudić, ${ }^{[b]}$ and Jin-Chong Tan*[a]
}

We propose a facile one-pot mechanochemical strategy for the encapsulation of drug guest molecules (e.g. 5-fluorouracil, caffeine, and aspirin) in MIL-100 (Fe) as the porous host framework. We reveal the modulating effect of 5-FU and caffeine, giving highly crystalline MIL-100 (Fe), whereas aspirin led to the formation of an amorphous MOF phase. Inelastic neutron scattering (INS) was used to probe the intermolecular interactions underpinning the drug@MIL-100 assemblies, where strong guesthost interaction was achieved via this eco-friendly approach.

Amongst the large family of nano-sized structures, metal-organic frameworks (MOFs) have been widely investigated as novel materials for drug delivery and theranostic applications. ${ }^{1}$ In this class of hybrid materials, the iron (III) carboxylate MOFs are promising candidates owing to their biologically and environmentally friendly nature, ${ }^{2}$ high accessible porosity and specific surface areas. In particular, MIL-100 (Fe) [MIL = Materials of Institute Lavoisier] exhibits a highly ordered structure with large nanoscale pores $(\sim 3 \mathrm{~nm})$, presenting numerous coordinatively unsaturated metal sites (CUS) that can act as strong binding sites for coordination with therapeutic drug molecules. ${ }^{3}$ Due to the intrinsic complexity of this three-dimensional framework, the synthesis of MIL-100 (Fe) conventionally entails long reactions under harsh conditions i.e. high pressure/temperature in boiling organic solvents combined with the use of harmful mineralizing agents such as hydrofluoric acid (HF) and concentrated nitric acid $\left(\mathrm{HNO}_{3}\right){ }^{4}$ Thus, environmentally friendly and scalable methods are desired for practical applications. ${ }^{5}$

To reduce the environmental impact of the synthesis and improve the biocompatibility of the product, many efforts have been put into engineering greener and faster synthetic methods for the fabrication of MOF structures. ${ }^{6}$ Specifically, new methodologies for the preparation of MIL-100 (Fe) have included solvent-free mechanochemical methods and water-based approaches,, 7 free from the noxious mineralizing agents that could affect the MOF applicability in the biomedical field. ${ }^{8}$ Developing new synthetic strategies are an enduring challenge due to the high complexity of the skeletal and topological structure of large-pore MOFs such as MIL-100 (Fe).

In this work, we present the application of a mechanochemical in situ method for the fabrication of a drug-encapsulated MIL-100 (Fe) system, designated as “drug@MIL-100", under ambient

\footnotetext{
a. Multifunctional Materials \& Composites (MMC) Lab, Department of Engineering Science, University of Oxford, Parks Road, Oxford OX1 3PJ, UK.

E-mail: jin-chong.tan@eng.ox.ac.uk

b.STFC Rutherford Appleton Laboratory, ISIS Neutron and Muon Source, Chilton, Didcot OX11 0QX, UK

tElectronic Supplementary Information (ESI) available: [Materials synthesis methods, materials characterization techniques, INS spectra, electron micrographs, adsorption isotherms, TGA and FTIR spectra.]. See DOI: 10.1039/x0xx00000x
}

conditions. We report the 'modulating' effect of the 'guest' drug molecules: 5 -fluorouracil (5-FU) and caffeine (CAF) on facilitating the formation of highly crystalline MIL-100 (Fe), akin to the role of a conventionally applied mineralizer. Conversely, aspirin (ASP) leads to the fabrication of an amorphous MOF phase. These are model drug molecules that have been selected based on continuous interest in their therapeutic effects, which, despite ongoing efforts, remain underrealized due to various practical limitations e.g. low bioavailability and side effects when orally administered. ${ }^{9}$ We studied the vibrational dynamics of the drug@MOF systems via inelastic neutron scattering (INS at TOSCA spectrometer), ${ }^{10}$ gaining insights into guest-host interactions through the collective vibrational modes. Understanding the underpinning molecular interactions is key for controlling the release of confined drug (guest) molecules from the MOF (host) carriers. ${ }^{11}$ We demonstrate the feasibility of this method as a facile one-pot self-assembly strategy, and shed new light on environmentally green approaches towards the fabrication of the MIL-100 (Fe) framework.

Fig. 1a schematically illustrates the in situ encapsulation process through mechanochemistry. All bulk reactants (i.e. organic linker $\mathrm{H}_{3} \mathrm{BTC}$, iron nitrate, and drug molecules) were manually ground in a mortar and pestle; details of the synthetic routes are given in the Supplementary Information (SI). The crystallinity of the resulting materials was examined via powder X-ray diffraction (PXRD) (Fig. 1b). MIL-100 (Fe) together with 5-FU and caffeine loaded systems were successfully synthesized. For 5-FU@MIL-100 and CAF@MIL-100, the main Bragg diffraction peaks below $2 \vartheta=5^{\circ}$ are apparent and a highly ordered structure is confirmed. Conversely, in ASP@MIL-100 the sharp Bragg peaks at low diffraction angles are completely absent, substituted by broad peaks located around $2 \vartheta=4-5^{\circ}, 6-8^{\circ}$, and $10-12^{\circ}$, indicating the amorphous character of this sample. We further assessed the structural differences between these samples by analysing the relative peak intensity of the (022):(357) planes (i.e. the two most intense diffraction peaks in MIL-100 (Fe) pattern) as a function of drug molecule present during the grinding step of the synthesis. Complementarily, the full width at half maximum (FWHM) values of the (022) peak were also calculated (Fig. S1, SI) and are accompanied by the non-normalized PXRD patterns (Fig. S2, SI).

By carefully contrasting between the as-synthesized MIL-100 (Fe) and its drug-loaded counterparts, two conclusions can be drawn. First, from Fig. $1 \mathrm{~b}$, it is possible to detect the deficiencies in the crystalline structure of MIL-100 (Fe). The absence of the diffraction peak at $2 \vartheta=3.4^{\circ}$, the small relative intensity of the (022):(357) planes (i.e. ratio of 1:0.84), and the slight broadness of the (022) peak (Fig. S1) all point to the partial formation of the crystalline framework. Likewise, the reduced relative intensity of the Bragg peaks at the small diffraction angles $\left(2 \vartheta<5^{\circ}\right)$ implies a weak long-range ordering of the MIL-100 (Fe) structure (Fig. S2). Fig. S3 shows the attenuated total reflectance Fourier transform infrared 
(a)

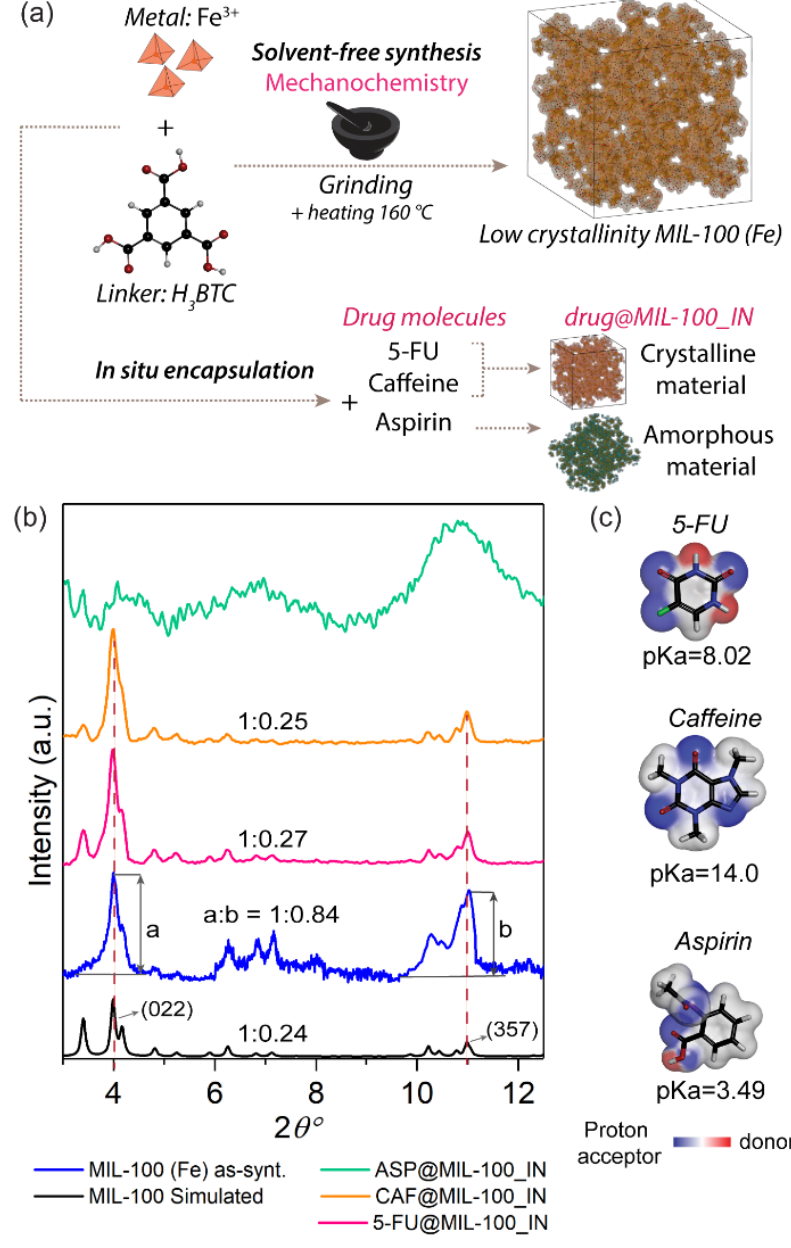

Fig. 1 Mechanochemical encapsulation and structural characterization of MIL-100 (Fe) and drug@MOF composites. (a) Schematic summarizing the manual grinding process used to fabricate the drug@MIL-100_IN samples (the suffix "IN" denotes guest encapsulation by in situ approach) and the respective effect of the different drug molecules on the framework crystallinity. (b) Normalized PXRD patterns of MIL-100 (Fe) and drug@MIL-100 assemblies showing the ratios between diffraction planes to assess the material crystallinity. The ratio was not computed for the aspirin-loaded system due to the amorphous nature of this sample. (c) Guest molecules of 5-FU, caffeine, and aspirin, highlighting their proton acceptor and donor sites. Colour scheme: iron in orange, carbon in black, oxygen in red, hydrogen in white, nitrogen in purple, and fluorine in green.

(ATR-FTIR) spectra of all the samples. The typical vibrational bands of MIL-100 (Fe) (i.e. C-H stretching in the organic linker at $\sim 707 \mathrm{~cm}^{-1}$ and $\sim 760 \mathrm{~cm}^{-1}$, O-C-O stretching and $\mathrm{O}-\mathrm{H}$ bending at $\sim 1371 \mathrm{~cm}^{-1}$ and $\sim 1441 \mathrm{~cm}^{-1}$, and $\mathrm{C}=\mathrm{O}$ stretching in the carboxylic group at $\left.\sim 1623 \mathrm{~cm}^{-1}\right)^{12}$ were detected in all samples, confirming the retainment of chemical bonds integrity. The presence of the vibrational band at $\sim 1720 \mathrm{~cm}^{-1}$ in the spectrum of the MIL-100 (Fe), assigned to the stretching of carboxyl group present in the acidic form of the organic linker (i.e. $\mathrm{H}_{3} \mathrm{BTC}=$ benzene1,3,5-tricarboxylic acid), ${ }^{4 c}, 13$ indicates the presence of remaining protonated organic ligand in the structure of MIL-100 (Fe). Because the full deprotonation of $\mathrm{H}_{3} \mathrm{BTC}$ is needed to yield a three-dimensional open framework with high crystallinity, the incomplete deprotonation of the organic ligand may hinder the complete self-assembly of the secondary building units (SBU). We reasoned that this has resulted in the partially formed (defective) MIL-100 (Fe) mesocages (Fig. 1a) with a lower crystallinity. ${ }^{14}$
Secondly, as revealed by the PXRD patterns in Fig. 1b, via the in situ strategy, highly crystalline 5-FU@MIL-100_IN (ratio 1:0.27) and CAF@MIL-100_IN (ratio 1:0.25) samples were obtained, while the presence of aspirin resulted in the production of an amorphous ASP@MIL-100_IN product. Contrasting the ATR-FTIR data of the MIL-100 (Fe) and the drug@MIL-100 systems, the sharpening of the band at $\sim 1355 \mathrm{~cm}^{-1}$, assigned to the stretching of the carboxylate groups present in the BTC organic linker, was observed. The change was characterized by the decrease in FWHM of this band (Fig. S3, SI). We have observed the sharpening of $\sim 42 \%$ and $~ 40 \%$ for 5-FU@MIL-100_IN and CAF@MIL-100_IN, respectively, suggesting the increase in the structural symmetry of the MIL-100 (Fe) host upon the presence of drug molecules. ${ }^{15}$ Conversely, the ASP@MIL-100_IN system remained virtually unchanged (sharpening of $\sim 4 \%$ ). The changes to the band at $1355 \mathrm{~cm}^{-1}$ were accompanied by the decline in intensity of the band at $\sim 1720 \mathrm{~cm}^{-1}$. This was quantified by the reduction in the integrated spectral area of this vibrational peak for both 5-FU@MIL-100_IN and ASP@MIL-100_IN systems (Fig. S3, SI). Such an analysis was not carried out for CAF@MIL-100_IN due to superposition of the BTC linker band and a vibrational band of caffeine (guest) in the same spectral range. The decline in the intensity of this band implies the decrease in the amount of protonated linker present in the sample.

As the intensity of the diffraction peaks is attributed to the periodic atomic arrangement averaged over the whole polycrystalline sample, ${ }^{16}$ the total increase in intensity observed in the PXRD patterns of the drug@MIL-100 systems in comparison to the as-synthesized MIL-100 (Fe) (Fig. S2) indicates the increased long-range ordering of the host MIL-100 (Fe) crystals when 5-FU or caffeine were present during mechanochemical synthesis. As shown in Fig. 1c, 5-FU, caffeine, and aspirin are molecules possessing proton $\left(\mathrm{H}^{+}\right)$acceptor atoms (i.e. oxygen, nitrogen, and fluorine), which can potentially facilitate the deprotonation of the $\mathrm{H}_{3} \mathrm{BTC}$ organic ligand and aid the formation of MIL-100 (Fe) cages. pKa values are also presented in Fig. 1c, indicating that among the drug molecules, 5-FU and caffeine are the most likely molecules to act as proton acceptors. Therefore, via the in situ encapsulation process, 5-FU and caffeine function as 'modulators' by aiding the deprotonation of the organic linkers. This results in formation of highly crystalline MIL-100 (Fe) frameworks, which simultaneously confine the drugs within the mesocages. The templating effect of caffeine on the formation of 3D structures has also been reported. ${ }^{9 b}$ Meanwhile, the high acidity of aspirin molecules ( $\mathrm{pKa}=3.49$ ) compromises the establishment of the long-range periodicity of the framework and results in the obtained amorphous phase. Strong broadening of the (022) peak was noticed when aspirin was present during the in situ synthesis (Fig. S1, SI). This is due to the competition between $\mathrm{H}_{3} \mathrm{BTC}$ and aspirin for the coordination with the iron ions, resulting in the formation of an amorphous violet aspirin-iron complex known as tetraaquosalicylatroiron (III) complex (Fig. S4, SI). Vibrational bands attributed to the complex have been identified in the ATR-FTIR spectrum of ASP@MIL-100_IN as shown in Fig. S5 in the SI, 

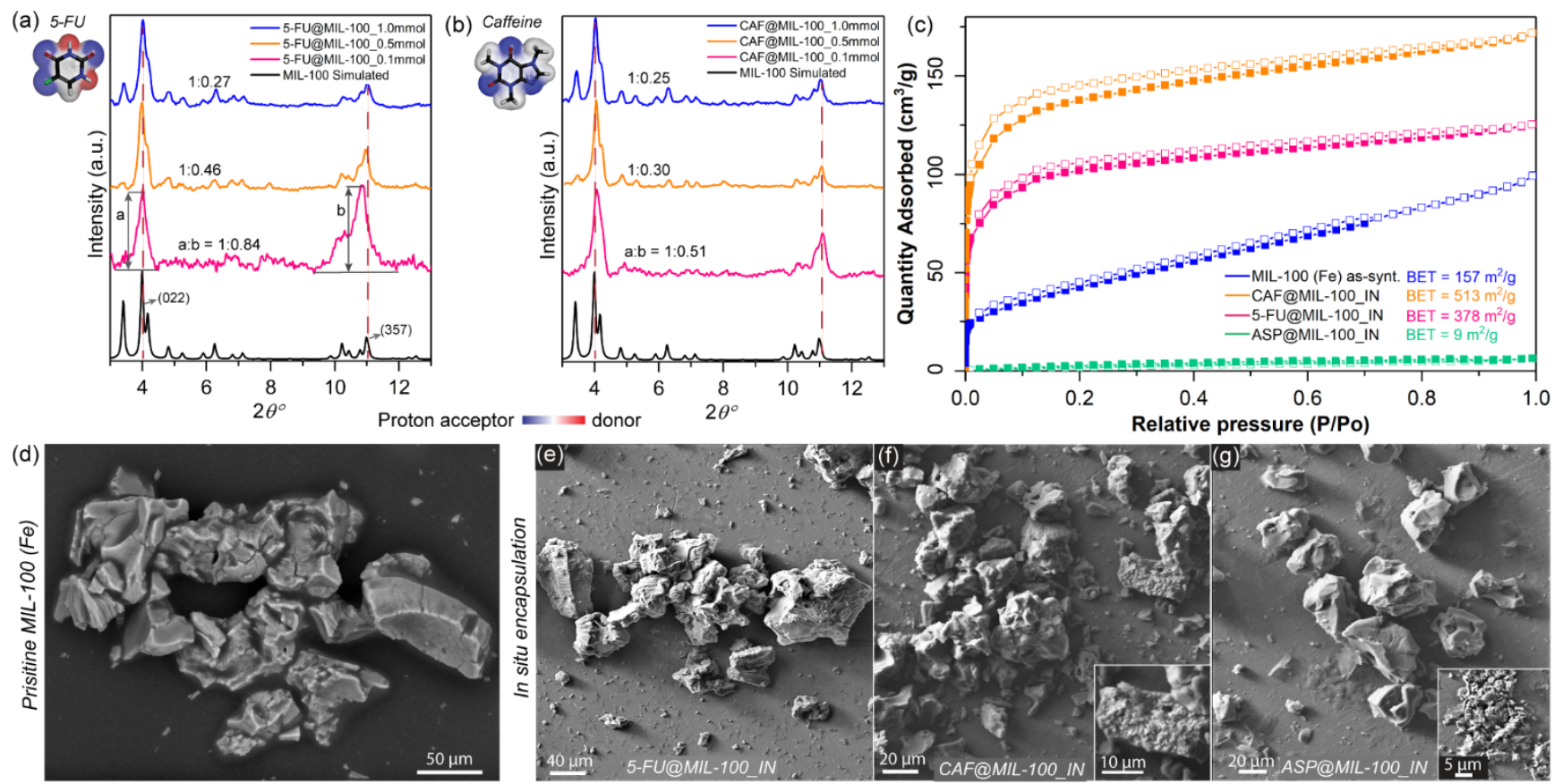

Fig. 2 Normalized PXRD patterns of (a) 5-FU@MIL-100_IN and (b) CAF@MIL-100_IN samples, showing the effect of the drug:linker ratio (i.e. x mmol of drug per 1.0 mmol of $\mathrm{H}_{3} \mathrm{BTC}$ ) used during the synthesis on the final crystallinity of the resulting material. The relative intensity of PXRD peaks was determined from the ratio of (022):(357). (c) Nitrogen adsorption (filled symbols) and desorption (empty symbols) isotherms of MIL-100 (Fe) and drug-loaded counterparts. SEM images of (d) MIL-100 (Fe) and drug@MIL-100 systems:

(e) 5-FU@MIL-100_IN, (f) CAF@MIL-100_IN, (g) ASP@MIL-100_IN. Colour code: O in red, C in black, H in grey, N in navy blue, F in green.

confirming the complex formation. The nature of these vibrations has been characterized further by density functional theory (DFT) calculations and compared with the experimental spectrum in Fig. S5 and Table S4, in the SI.

To further assess the modulating effect of 5-FU and caffeine, we have investigated the influence of the drug: $\mathrm{H}_{3} \mathrm{BTC}$ molecular ratio on the crystallinity of the resulting material. To accomplish the complete deprotonation of the organic linker, the dissociation of 3 hydrogen atoms for each $\mathrm{H}_{3} \mathrm{BTC}$ molecule is necessary. In principle, both 5-FU and caffeine have the potential to accept 3 protons each, but pKa values indicate that caffeine has a higher potential to act as a proton acceptor. Normalized PXRD patterns in Fig. 2 show the effect on the crystallinity of the final samples when different amounts of 5FU (Fig. 2a) and caffeine (Fig. 2b) were added during the grinding process. As the amount of drug molecules increases (e.g. from 0.1 to $1.0 \mathrm{mmol}$ of $\mathrm{drug} / \mathrm{mmol}$ of $\mathrm{H}_{3} \mathrm{BTC}$ ), an evident increase in the relative intensity of the diffraction peaks was observed. Even with only $0.1 \mathrm{mmol}$ of caffeine per mmol of $\mathrm{H}_{3} \mathrm{BTC}$, improvements in crystallinity of CAF@MIL-100_IN were detected when compared to MIL-100 (Fe). With $1.0 \mathrm{mmol}$ of both 5-FU and caffeine per mmol of $\mathrm{H}_{3} \mathrm{BTC}$, highly crystalline material has been obtained. We have further assessed the degree of crystallinity of these samples by measuring the FWHM of the (022) peak (Fig. S6, SI), which showcases the increase in the crystallinity of the MIL-100 (Fe) host. As anticipated, caffeine presents a slightly better modulator performance due to its relatively higher pKa (i.e. marginally higher proton acceptor potential).

Nitrogen sorption isotherms (Fig. 2c) and scanning electron microscopy (SEM) images (Fig. 2d-g) were used to further characterize the drug@MIL-100 system. SEM images, employed to examine the morphology of the samples, show no signs of alterations upon drug encapsulation. The formation of the observed MIL-100 (Fe) crystal aggregates seems to be a result of the grinding process, culminating in the non-uniform particles size range observed (i.e. 2-100 $\mu \mathrm{m}$ ). The amorphous ASP@MIL-100_IN product also presented itself in an aggregated form, with an equally large distribution of particle sizes.

Due to the lack of noticeable morphological differences between the amorphous and crystalline phases, Brunauer-Emmett-Teller (BET) surface areas of MIL-100 (Fe) and drug-loaded counterparts were determined from the nitrogen sorption isotherms to quantify the samples porosity (Fig. 2c). The isotherms were also used to determine the level of guest encapsulation, which were further quantified by thermogravimetric analysis (TGA) (Fig. S7 and Table S2, SI). The surface area of the MIL-100 (Fe) $\left(157 \mathrm{~m}^{2} \mathrm{~g}^{-1}\right)$ was largely reduced when compared with values of other reported mechanochemically synthesized MIL-100 (Fe) samples (Table S3, ESI). 1717 The enhanced development of the host framework structure due to the modulating effect of 5-FU and caffeine also had a visible impact on the material surface areas, being $378 \mathrm{~m}^{2} \mathrm{~g}^{-1}$ and $513 \mathrm{~m}^{2} \mathrm{~g}^{-1}$ for 5-FU@MIL-100_IN and CAF@MIL-100_IN, respectively. It is important to note that the surface areas of these drug@MIL-100 systems should not be directly compared to the ones presented in Table S3. This is because the improvement of MIL-100 (Fe) porous structure attributed to the modulating effect of the drug molecules occurs in parallel with its partial obstruction, occasioned by the drug molecules confinement. Finally, the isotherm shape and the significantly reduced surface area found in ASP@MIL-100_IN $\left(9 \mathrm{~m}^{2} \mathrm{~g}^{-1}\right)$ showcase the non-porous character of this drug-loaded amorphous material. For comparison, we have collected the PXRD pattern and ATR-FTIR spectrum of a commercial grade of MIL-100 (Fe), termed Basolite F300 (Fe-BTC). It 
displays broad PXRD peaks, suggesting an amorphous structure, but its vibrational spectrum remains intact (Fig. S8), in a similar fashion to ASP@MIL-100_IN. These observations imply that the aspirinloaded system retains the basic building blocks and short-range connectivity of the crystalline MIL-100 (Fe), but it lacks long-range periodic order, possibly being impeded by the formation of the tetraaquosalicylatroiron (III) complex.

We have determined the drug loadings of $20.4 \mathrm{wt} . \%$ (0.26 g/g MOF), $54.8 \mathrm{wt} . \% \quad$ (1.21 g/g MOF), and $9.8 \mathrm{wt} . \%$ (0.11 g/g MOF) for 5-FU@MIL-100_IN, CAF@MIL-100_IN, and ASP@MIL-100_IN, respectively. In Table S2, we compare the drug loading herein attained with previously reported results (see SI for further detail). The sizeable loading found for 5-FU and caffeine is a result of the advantageous formation of the host MIL-100 (Fe) cages around the guest drug molecules, made possible by the in situ encapsulation approach applied here. The low drug loading of aspirin is attributed to the formation of the aspirin-iron complex, which is mostly removed during the wash steps (see Fig. S4, SI).

The vibrational properties of MIL-100 (Fe) and drug@MIL-100 systems were characterized by inelastic neutron scattering (INS), and the results are presented in Fig. 3. ${ }^{18}$ Neutron scattering is a high-resolution spectroscopic technique leveraging the scattering of neutrons by the nuclei of condensed matter. ${ }^{19}$ The neutron is a highly sensitive probe to measure local changes in vibrational modes, especially the low energy phonons or terahertz $(\mathrm{THz})$ vibrations, which represent collective modes associated with the lattice dynamics of the framework structure. ${ }^{20}$ INS spectroscopy of the drug@MIL-100 system probes the dynamic properties of the drugencapsulated MIL-100 (Fe) samples, revealing the modulator effect of the guest drug molecules. The full spectra up to $\sim 60 \mathrm{THz}$ $\left(2000 \mathrm{~cm}^{-1}\right)$ are shown in Figs. S9-S11 in the SI.

Detailed comparison between the INS spectra of the in situ derived samples and the MIL-100 (Fe) reveals an overall lower spectral intensity of 5-FU and aspirin encapsulated MIL-100 (Fe) systems. This can be credited to two main factors. Firstly, the INS spectrum intensity at low frequency (i.e. low momentum transfer $(Q))$, as recorded on TOSCA, is proportional to the mean square displacement of the atoms from their equilibrium position. At higher frequency the intensity is suppressed by the Debye-Waller factor. ${ }^{21}$ On this basis, the decrease in the scattering intensity in the low energy region of the drug@MIL-100 systems, specifically for 5-FU@MIL-100_IN and ASP@MIL-100_IN, suggests the decrease of atomic motions. As the periodicity of the MIL-100 (Fe) host is enhanced, stronger constraints are imposed on the lattice modes.

Another factor contributing to the observed reduction of spectral intensity is the decrease in the total amount of hydrogen atoms present within the sample, resulting from the large incoherent neutron scattering cross-section of hydrogen (which is the largest amongst all known elements). ${ }^{19}$ In this case, the increase in the effective deprotonation of the organic linker as detected in ATR-FTIR measurements (Fig. S2), due to the modulating effect of 5-FU and caffeine, and the consequential reduction of the number of hydrogens present in the samples resulted in the decline of overall spectral intensity. For the amorphous ASP@MIL-100_IN system the decline can be linked to the formation of the aspirin-iron complex which reduces the overall number of $\mathrm{H}_{3} \mathrm{BTC}$ molecules within the sample. Additionally, despite its amorphous nature, this system still
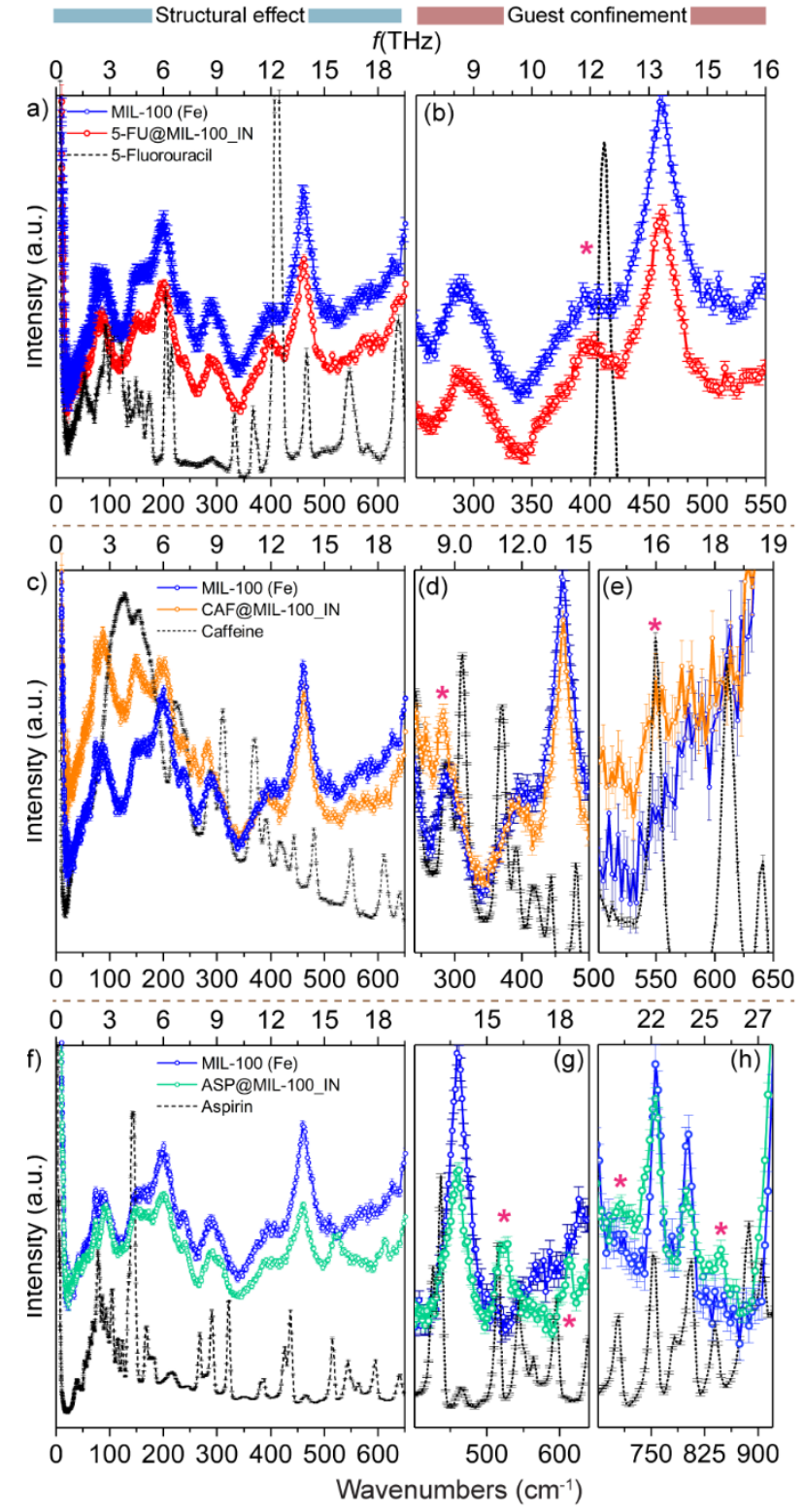

Fig. 3 INS spectra of (a-b) 5-FU@MIL-100_IN, (c-e) CAF@MIL-100_IN, and (f-h) ASP@MIL-100_IN, with magnified views of the drug peaks present in the drug@MIL-100 systems, highlighted with asterisks.

presents all the collective modes observed in the MIL-100 (Fe), akin to what was detected in the higher energy vibrational bands of the ATR-FTIR spectra (Fig. S2).

Analysis of the zoom-ins in Fig. 3 shows that in all drug@MIL-100 samples, drug vibrational peaks (highlighted with asterisks) were observed. $A b$ initio DFT calculations further revealed details of the specific drug molecule vibrations (Figs. S12-S14 and Table S4, SI). Scrutiny of the INS spectra provides a deeper insight into the dynamics of the guest-host interaction. The higher intensity observed in the spectra of CAF@MIL-100_IN below $9 \mathrm{THz}\left(300 \mathrm{~cm}^{-1}\right)$ is linked to the combined scattering of the MOF host and the caffeine guest molecule, specifically resulting from the high guest loading ( $\sim 5 \mathrm{wt} . \%)$ of this system. Vibrational frequency shifts in the guest vibrational modes after confinement were detected in all samples (marked by asterisks in Fig. 3 insets), indicating the presence of constraints to the free motions of the drug molecules due to pore 
confinement. This effect is clear in the shift and suppression of the vibrational mode of 5-FU at $\sim 12.3 \mathrm{THz}\left(\sim 410 \mathrm{~cm}^{-1}\right)$, the caffeine mode at $\sim 8.6 \mathrm{THz}\left(\sim 288 \mathrm{~cm}^{-1}\right)$, and the aspirin modes at $\sim 15.4 \mathrm{THz}$ $\left(\sim 514 \mathrm{~cm}^{-1}\right)$ and $\sim 19.2 \mathrm{THz}\left(\sim 641 \mathrm{~cm}^{-1}\right)$ in the spectra of the 5-FU@MIL-100_IN, CAF@MIL-100_IN, and ASP@MIL-100_IN, respectively. The origin of each of these vibrations has been detailed in Table S5, SI. Based on the INS spectra and on the electrostatic potential maps (ESP) of the drug molecules (Fig. S15, SI) we can conclude that 5-FU, caffeine, and aspirin form strong Fe-O coordination with the CUS of the MOF, conferred by the in situ encapsulation method applied. This approach is advantageous against the conventional post-synthetic drug encapsulation approach (by immersion in a drug solution) in which the drug/solvent competition for the coordinatively unsaturated binding site can lead to a weaker guest-host interaction. ${ }^{22}$

In summary, we have demonstrated the use of a facile mechanochemical method to achieve pore confinement of various 'guest' drug molecules within MIL-100 (Fe) pores. Notably this proofof-concept study shows how 5-FU and caffeine can function as 'modulators' leading to the fabrication of a highly crystalline MIL-100 (Fe) framework. As a counterexample, we have illustrated how aspirin molecules instigate the formation of an amorphous MIL-100 (Fe) phase. It is intriguing to see that benign modulators such as caffeine could potentially replace highly toxic mineralizers, such as HF commonly used in the preparation of MIL-100 (Fe). Our results elucidate a promising pathway towards an environmentally friendly approach to yield MIL-100 (Fe) crystals and guestencapsulated composites, thereby addressing the continuous search for low cost and scalable synthetic methods. Finally, using inelastic neutron scattering, we have established clear differences in the vibrational behaviour of the isolated drug molecules and the drug@MOF assemblies. Neutron vibrational spectroscopy reveals the intrinsic guest-host intermolecular interactions, pointing to the successful confinement of drug molecules due to the in situ mechanochemical grinding technique applied. The facile approach demonstrated herein can advance the future commercial potential of mesoporous MIL-100 materials.

B.E.S. thanks the Minas Gerais Research Foundation (FAPEMIG CNPJ n21.949.888/0001-83) for funding this research through a DPhil scholarship award. J.C.T. thanks the Engineering and Physical Sciences Research Council (EPSRC) Grant No. EP/ N014960/1 and European Research Council (ERC) Consolidator Grant under Grant Agreement 771575 (PROMOFS) for funding. We acknowledge the ISIS Neutron and Muon Source for the award of Beamtime No. RB1910059 during which the INS experiments were performed on the TOSCA spectrometer. ${ }^{18}$ We are grateful to the Research Complex at Harwell (RCaH) for the provision of advanced materials characterization facilities.

\section{Conflicts of interest}

There are no conflicts to declare

\section{Notes and references}

1. (a) Y. Liu, Y. L. Zhao and X. Y. Chen, Theranostics., 2019, 9, 31223133; (b) S. Rojas, T. Baati, L. Njim, L. Manchego, F. Neffati, N.
Abdeljelil, S. Saguem, C. Serre, M. F. Najjar, A. Zakhama and P. Horcajada, J. Am. Chem. Soc., 2018, 140, 9581-9586.

2. M. A. Simon, E. Anggraeni, F. E. Soetaredjo, S. P. Santoso, W. Irawaty, T. C. Thanh, S. B. Hartono, M. Yuliana and S. Ismadji, Sci. Rep., 2019, 9, 16907.

3. A. García Márquez, A. Demessence, A. E. Platero-Prats, D. Heurtaux, P. Horcajada, C. Serre, J.-S. Chang, G. Férey, V. A. de la Peña-O'Shea, C. Boissière, D. Grosso and C. Sanchez, Eur. J. Inorg. Chem., 2012, 2012, 5165-5174.

4. (a) B. Yuan, X. Wang, X. Zhou, J. Xiao and Z. Li, Chem. Eng. J., 2019, 355, 679-686; (b) L. Han, H. Qi, D. Zhang, G. Ye, W. Zhou, C. M. Hou, W. Xu and Y. Y. Sun, New J. Chem., 2017, 41, 13504-13509; (c) N. M. Mahmoodi, J. Abdi, M. Oveisi, M. Alinia Asli and M. Vossoughi, Mater. Res. Bull., 2018, 100, 357-366.

5. M. Gaab, N. Trukhan, S. Maurer, R. Gummaraju and U. Muller, Microporous Mesoporous Mat., 2012, 157, 131-136.

6. (a) T. Stolar and K. Uzarevic, CrystEngComm, 2020, 22, 4511-4525; (b) B. Karadeniz, A. J. Howarth, T. Stolar, T. Islamoglu, I. Dejanović, M. Tireli, M. C. Wasson, S.-Y. Moon, O. K. Farha, T. Friščić and K. Užarević, ACS. Sustain. Chem. Eng., 2018, 6, 15841-15849.

7. Y.-K. Seo, J. W. Yoon, J. S. Lee, U. H. Lee, Y. K. Hwang, C.-H. Jun, P. Horcajada, C. Serre and J.-S. Chang, Microporous Mesoporous Mat., 2012, 157, 137-145.

8. Y. Fang, J. Wen, G. Zeng, F. Jia, S. Zhang, Z. Peng and H. Zhang, Chem. Eng. J., 2018, 337, 532-540.

9. (a) J. Hou, C. Li, L. Cheng, S. Guo, Y. Zhang and T. Tang, Drug. Dev. Ind. Pharm., 2011, 37, 1068-1075; (b) N. Liédana, P. Lozano, A. Galve, C. Téllez and J. Coronas, J. Mater. Chem. B, 2014, 2, 11441151; (c) B. Singco, L.-H. Liu, Y.-T. Chen, Y.-H. Shih, H.-Y. Huang and C.-H. Lin, Microporous Mesoporous Mat., 2016, 223, 254-260.

10. (a) S. F. Parker, F. Fernandez-Alonso, A. J. Ramirez-Cuesta, J. Tomkinson, S. Rudic, R. S. Pinna, G. Gorini and J. Fernández Castañon, J. Phys. Conf. Ser., 2014, 554, 012003; (b) R. S. Pinna, S. Rudić, S. F. Parker, J. Armstrong, M. Zanetti, G. Škoro, S. P. Waller, D. Zacek, C. A. Smith, M. J. Capstick, D. J. McPhail, D. E. Pooley, G. D. Howells, G. Gorini and F. Fernandez-Alonso, Nucl. Instrum. Meth. A, 2018, 896, 68-74.

11. B. E. Souza, L. Dona, K. Titov, P. Bruzzese, Z. Zeng, Y. Zhang, A. S. Babal, A. F. Moslein, M. D. Frogley, M. Wolna, G. Cinque, B. Civalleri and J. C. Tan, ACS Appl. Mater. Inter., 2020, 12, 5147-5156.

12. H. Lv, H. Zhao, T. Cao, L. Qian, Y. Wang and G. Zhao, J. Mol. Catal. AChem., 2015, 400, 81-89.

13. S. Lin, Z. Song, G. Che, A. Ren, P. Li, C. Liu and J. Zhang, Microporous Mesoporous Mat., 2014, 193, 27-34.

14. B. E. Souza, A. F. Möslein, K. Titov, J. D. Taylor, S. Rudić and J.-C. Tan, ACS Sustain. Chem. Eng., 2020, 8, 8247-8255.

15. C. Petit, Factors Affecting the Removal of Ammonia from Air on Carbonaceous Materials: Investigation of Reactive Adsorption Mechanism, Springer Science \& Business Media, New York, USA, 2012.

16. D. S. Sivia, Elementary scattering theory : for X-ray and neutron users, Oxford University Press, Oxford, 2011.

17. (a) J. Yang, X. Feng, G. Lu, Y. Li, C. Mao, Z. Wen and W. Yuan, Dalton Trans., 2018, 47, 5065-5071; (b) M. Samal, J. Panda, B. P. Biswal and R. Sahu, Cryst. Eng. Comm., 2018, 20, 2486-2490; (c) M. Klimakow, P. Klobes, A. F. Thünemann, K. Rademann and F. Emmerling, Chem. Mater., 2010, 22, 5216-5221.

18. ISIS TOSCA Beamtime data, https://doi.org/10.5286/ISIS.E.RB1910059, (accessed July 2020).

19. F. Fernandez-Alonso and D. L. Price, Neutron Scattering Applications in Biology, Chemistry, and Materials Science Preface, Academic Press, Kidlington 2017.

20. M. R. Ryder, B. Civalleri, T. D. Bennett, S. Henke, S. Rudic, G. Cinque, F. Fernandez-Alonso and J. C. Tan, Phys. Rev. Lett., 2014, 113, 215502.

21. A. Griffin and H. Jobic, J. Chem. Phys., 1981, 75, 5940-5943.

22. B. E. Souza, S. Rudic, K. Titov, A. S. Babal, J. D. Taylor and J. C. Tan, Chem. Comm., 2019, 55, 3868-3871. 